\title{
A Probabilistic Method to Determine Whether the Speed of Light Is Constant
}

\author{
Motohisa Osaka \\ Department of Basic Science, Nippon Veterinary and Life Science University, Tokyo, Japan \\ Email: osaka@nms.ac.jp
}

How to cite this paper: Osaka, M. (2019) A Probabilistic Method to Determine Whether the Speed of Light Is Constant. $A p$ plied Mathematics, 10, 51-59.

https://doi.org/10.4236/am.2019.102005

Received: January 16, 2019

Accepted: February 12, 2019

Published: February 15, 2019

Copyright (C) 2019 by author(s) and Scientific Research Publishing Inc. This work is licensed under the Creative Commons Attribution International License (CC BY 4.0).

http://creativecommons.org/licenses/by/4.0/

\begin{abstract}
Although the formula of mass-energy equivalence was derived from the hypothesis that the speed of light in free space is constant, conversely, the purpose of this research is to show that a method of probabilistically determining whether the speed of light is constant is derived from this formula. By considering the formula of mass-energy equivalence to be a function of the energy of an object moving at speed $V$, the probability density function (PDF) of the energy can be obtained using the inverse function of this formula, if the speed of light obeys a probability distribution. The main result is that the PDF of the energy diverges to infinity at a certain energy value regardless of the PDF of the speed of light. Thus, when the speed calculated from this value enters a certain range of the speed of light as $V$ increases stepwise from below $299,792,458 \mathrm{~m} / \mathrm{s}$, the PDF of the energy should increase abruptly. If not, then the speed of light is constant. This is the method of probabilistically determining whether the speed of light is constant. An experimental method is proposed to confirm this.
\end{abstract}

\section{Keywords}

Special Relativity, Light Speed, Mass-Energy Equivalence

\section{Introduction}

Albert Einstein published the theory of special relativity in 1905 [1]. The theory is on the relationship between space and time. One of its results is mass-energy equivalence: $E=m c^{2}$, where $E$ is the energy of an object when it is moving, $m$ is its mass while moving and $c$ is the speed of light. This is derived from two hypotheses. One is that the speed of light in free space is constant for all observers, regardless of their relative motion or of the motion of the light source. This hypothesis is generally considered verified by the Michelson-Morley experiment, 
which shows the differences between the speed of light in the direction of motion of the earth and that in different directions are within experimental errors [2]. This was supported by similar experiments with higher resolutions [3] [4]. However, these experiments do not show that the speed of light in free space is constant in all inertial systems. A team at University of Glasgow reported that photon group velocity was reduced using time-correlated photon pairs and that the delay was several micrometers over a propagation distance of the order of 1 $\mathrm{m}$ [5]. Although they showed that adding spatial structure to an optical beam of single photons reduced the speed of light, the significance of their study was considered to be limited. The findings do not affect the formula of energy-mass equivalence because $c$ in the formula is still regarded as the maximum speed of all objects in free space. However, the question is whether the maximum speed is constant. This study presents a probabilistic method derived from $E=m c^{2}$ to determine whether the speed of light is constant.

\section{Mathematical Steps}

It assumes that the speed of light obeys a probability distribution.

The formula $E=m c^{2}$ is also expressed as

$$
E=\frac{m_{0}}{\sqrt{1-\left(\frac{V}{c}\right)^{2}}} c^{2},
$$

where $m_{0}$ is the rest mass of the object, and $V$ is its speed; $V<c$.

The assumptions are:

1) $m_{0}$ and $V$ are constant.

2) $c$ obeys a probability distribution between $c_{a}$ and $c_{b}$; $c_{a}<c_{b}$. Two probability distributions are adopted: a uniform distribution and a triangular distribution.

Then, the probability density function (PDF) of $E$ is calculated by obtaining the inverse function of $E$ as follows.

Step 1: Determining the inverse function of $E$

As $0<V / c<1, V / c$ is defined by $\sin \theta(0<\theta<\pi / 2)$.

From (1),

$$
E=\frac{m_{0} V^{2}}{\cos \theta-\cos ^{3} \theta} .
$$

Then, $\cos \theta$ is represented as $y$.

$$
c=\frac{V}{\sqrt{1-y^{2}}} .
$$

Representing $m_{0} V^{2}$ as $P_{0}$, the following third-order equation for $y$ is obtained from (2):

$$
f(y)=y^{3}-y+\frac{P_{0}}{E}=0 .
$$

A positive value of $E$ results from any value of $\theta$ on the interval $0<\theta<\pi / 2$. In 
other words, at least one of the three roots of $f(y)$ is between 0 and 1 . As the roots of $f(y)$ are intersections of $g(y)=y^{3}-y=y(1-y)(1+y)$ and $h(y)=$ $-P_{0} / E$, all three roots are real. Two of them are between 0 and 1 and the remaining root is negative. The two roots between 0 and 1 are denoted as $y_{1}$ and $y_{2} ; y_{1} \leq$ $y_{2}$. As $0<y<1$, the negative root is neglected. Since $y_{1}$ and $y_{2}$ are determined by $E$, it is necessary to find out whether the inverse function of $E, c \equiv h(E)$, is a two-valued or single-valued function.

Step 2: Determining whether $E$ is a two-valued or singled-valued function

From (1), $E$ has the minimum $\frac{3 \sqrt{3}}{2} P_{0}\left(P_{0}=m_{0} V^{2}\right)$ at $c_{m} \equiv \frac{\sqrt{6}}{2} V$. Three cases are examined on the basis of whether $c_{m}$ is between $c_{a}$ and $c_{b}$.

Case 1: $c_{m} \leq c_{a}$

Then $V \leq \frac{\sqrt{6}}{3} C_{a}$. As $E$ increases monotonically between $c_{a}$ and $c_{b}$,

$$
\frac{V}{\sqrt{1-y_{1}^{2}}}<c_{a} \text { and } c_{a} \leq \frac{V}{\sqrt{1-y_{2}^{2}}} \leq c_{b} .
$$

Therefore, only $y_{2}$ is accepted. In this case $c=h(E)$ is a single-valued function (Figure 1(a)).



(a)

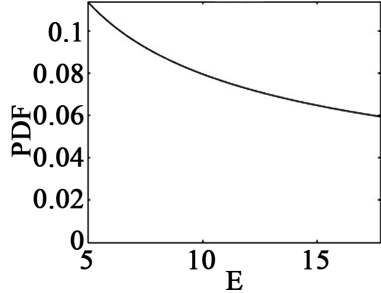

(b)

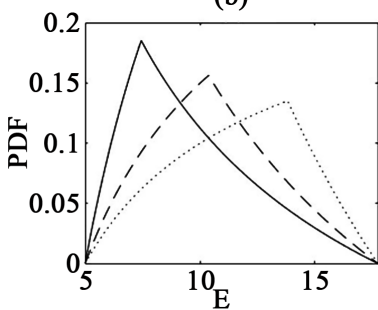

(c)

Figure 1. Case of $c_{m} \leq c_{a}$. The object speed and limits of $c$ are set to $V=0.5, c_{a}=2.2$ and $c_{b}=4.2$. Then, $c_{m} \approx 0.6124$. (a) Inverse function of $E$ for $c$, (b) Probability distribution of $E$ when $c$ obeys the uniform distribution; (c) Probability distribution of $E$ for three cases: the $c$-value of the vertex of the triangular probability distribution of $c$ is 2.7 (solid line), 3.2 (dashed line) or 3.7 (dotted line). The probability distribution of $E$ is triangle-like with the vertex moving rightward with the probability distribution of $c$. 
Case 2: $c_{a}<c_{m}<c_{b}$

Then $\frac{\sqrt{6}}{3} C_{a}<V<\frac{\sqrt{6}}{3} C_{b}$. As $E$ is parabolic and downward convex between $c_{a}$ and $c_{b}$, the following relationship occurs:

$$
c_{a}<\frac{V}{\sqrt{1-y_{1}^{2}}}<\frac{V}{\sqrt{1-y_{2}^{2}}}<c_{b} .
$$

Both $y_{1}$ and $y_{2}$ are accepted and $c=h(E)$ is a two-valued function (Figure 2(a)). Otherwise, the following occurs:

$$
\frac{V}{\sqrt{1-y_{1}^{2}}}<c_{a} \text { or } c_{b}<\frac{V}{\sqrt{1-y_{2}^{2}}} .
$$

Then $c=h(E)$ is single-valued function (only $y_{2}$ is accepted in Figure 2(a)).

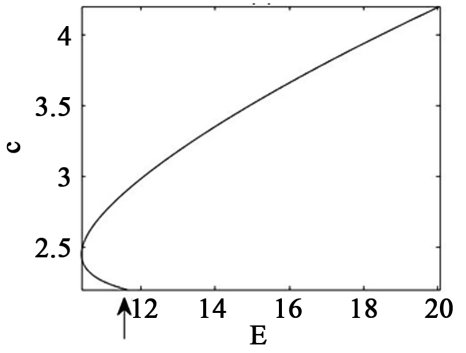

(a)

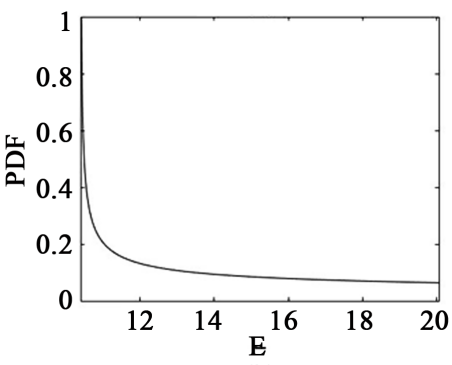

(b)

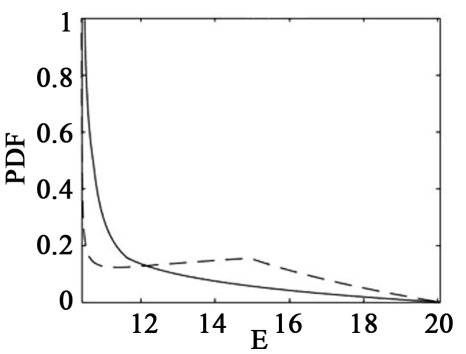

(c)

Figure 2. Case of $c_{a}<c_{m}<c_{b}$. The object speed and limits of $c$ are set to $V=2, c_{a}=2.2$ and $c_{b}=4.2$. Then, $c_{m} \approx 2.4995$. (a) The inverse function of $E$ is a two-valued function between minimum $E$ and the value marked with an arrow and a one-valued function between the marked value and maximum $E$; (b) Probability distribution of $E$ when $c$ obeys the uniform distribution; (c) Probability distribution of $E$ for two cases: the $c$-value of the vertex of the triangular probability distribution of $c$ is less than $c_{m},\left(2.3<c_{m}\right)$ (solid line), and larger than $c_{m}$, $\left(3.7>c_{m}\right)$, (dashed line). In the former, the PDF of $E$ decreases monotonically. In the latter, it has a vertex. If $c_{a}<c_{m}<c_{b}$, the probability density of $E$ is maximum at minimum $E$ in either distribution of $c$. 
Case 3: $c_{m} \geq c_{b}$

Then $V \geq \frac{\sqrt{6}}{3} C_{b}$. As $E$ decreases monotonically between $c_{a}$ and $c_{b}$,

$$
c_{a} \leq \frac{V}{\sqrt{1-y_{1}^{2}}} \leq c_{b} \text { and } c_{b}<\frac{V}{\sqrt{1-y_{2}^{2}}} .
$$

Therefore, only $y_{1}$ is accepted. In this case $c=h(E)$ is also a single-valued function (Figure 3(a)).

Step 3: Calculation of PDF of $E$

From the PDF of $c, f_{c}(c)$, and the inverse function of $E, c=h(E)$, the PDF of the random variable $E, f_{E}(E)$, can be obtained as

$$
f_{E}(E)=f_{c}[h(E)]\left|\frac{\mathrm{d}}{\mathrm{d} E} h(E)\right|
$$

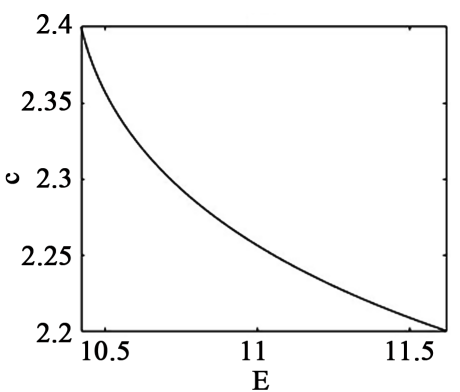

(a)

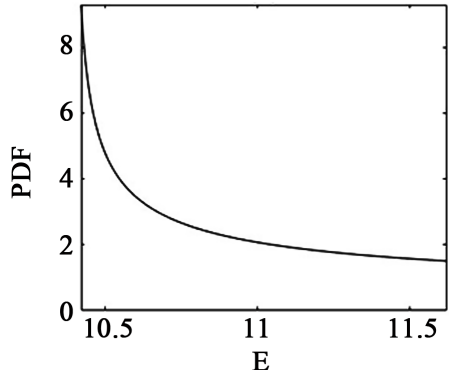

(b)

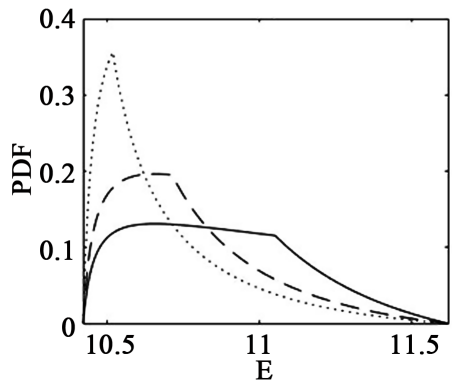

(c)

Figure 3. Case of $c_{m} \geq c_{b}$. The object speed and limits of $c$ are set to $V=2, c_{a}=2.2$ and $c_{b}=$ 2.4. Then, $c_{m} \approx 2.4995$. (a) Inverse function of $E$ for $c$, (b) Probability distribution of $E$ when $c$ obeys the uniform distribution; (c) Probability distribution of $E$ for three cases: the $c$-value of the vertex of the triangular probability distribution of $c$ is 2.25 (solid line), 2.3 (dashed line) or 2.35 (dotted line). The probability distribution of $E$ changes from trapezoid-like shape to triangle-like shape as the vertex of the probability distribution of $c$ moves rightward. 
where $\left|\frac{\mathrm{d}}{\mathrm{d} E} h(E)\right|$ is the Jacobian of the transformation. The absolute value should be taken since the PDF must be positive:

$$
\left|\frac{\mathrm{d} c}{\mathrm{~d} E}\right|=\left|\frac{\mathrm{d}}{\mathrm{d} E} h(E)\right|=\left|\frac{\mathrm{d} c}{\mathrm{~d} \theta}\right|\left|\frac{\mathrm{d} \theta}{\mathrm{d} E}\right|
$$

From $c=V / \sin \theta$ and $y=\cos \theta$,

$$
\left|\frac{\mathrm{d} c}{\mathrm{~d} \theta}\right|=\sqrt{\frac{P_{0}}{m_{0}}}\left|-\frac{\cos \theta}{1-\cos ^{2} \theta}\right|=\sqrt{\frac{P_{0}}{m_{0}}} \frac{y}{1-y^{2}} .
$$

From (2) and $y=\cos \theta$,

$$
\left|\frac{\mathrm{d} \theta}{\mathrm{d} E}\right|=\frac{P_{0}}{E^{2}} \frac{1}{\sin \theta\left|1-3 \cos ^{2} \theta\right|}=\frac{P_{0}}{E^{2}} \frac{1}{\sqrt{1-y^{2}}\left|1-3 y^{2}\right|} .
$$

From (10), (11) and (12),

$$
\left|\frac{\mathrm{d} c}{\mathrm{~d} E}\right|=\left|\frac{\mathrm{d}}{\mathrm{d} E} h(E)\right|=\frac{P_{0} \sqrt{P_{0}}}{\sqrt{m_{0}}} \frac{y}{\left(1-y^{2}\right) \sqrt{1-y^{2}}\left|1-3 y^{2}\right|} \frac{1}{E^{2}} .
$$

From (9) and (13), the PDF of $E$ can be obtained:

$$
\begin{aligned}
f_{E}(E) & =f_{c}[h(E)]\left|\frac{\mathrm{d}}{\mathrm{d} E} h(E)\right| \\
& =f_{c}[h(E)] \frac{P_{0} \sqrt{P_{0}}}{\sqrt{m_{0}}} \frac{y}{\left(1-y^{2}\right) \sqrt{1-y^{2}}\left|1-3 y^{2}\right|} \frac{1}{E^{2}}
\end{aligned}
$$

As $0<y<1, f_{E}(E)$ diverges to infinity at $y=\frac{1}{\sqrt{3}}$. From (3), $c=\frac{\sqrt{6}}{2} V$. This value of $c$ is equal to $c_{m}$. Then $E=\sqrt{3} m_{0} c_{m}^{2}$.

Step 4: Setting of parameters

As the value of $m_{o}$ has no qualitative effect on the relationship between $E$ and $c, m_{o}$ is set equal to 1 . The values of $V, c_{a}$ and $c_{b}$ are set arbitrarily, depending on whether $c_{m}$ is within the range of $c . c_{a} \leq c \leq c_{b}$.

\section{Results}

Case 1: $c_{m} \leq c_{a}$

The object speed and limits of $c$ are set to $V=0.5, c_{a}=2.2$ and $c_{b}=4.2$. Then $c_{m} \approx 0.6124$. Figure 1 (a) shows the relationship between $E$ and $c$. The inverse function of $E$ is a single-valued monotonically increasing function. Figure 1(b) shows the PDF of $E$, which decreases monotonically when $c$ obeys the uniform distribution. Figure 1(c) shows three cases: the $c$-value of the vertex of the triangular probability distribution of $c$ is $2.7,3.2$ or 3.7 .

Case 2: $c_{a}<c_{m}<c_{b}$

The object speed and limits of $c$ are set to $V=2, c_{a}=2.2$ and $c_{b}=4.2$. Then $c_{m}$ $\approx 2.4495$. Figure 2 (a) shows the relationship between $E$ and $c$. The inverse function of $E$ is a two-valued function between minimum $E$ and a certain value marked with an arrow and a single-valued function between the marked value 
and maximum $E$. In Figure 2(b), the PDF of $E$ decreases monotonically when $c$ has the uniform probability distribution. Figure 2(c) shows two cases: the $c$-value of the vertex of the triangular probability distribution of $c$ is less than $c_{m}$, $\left(2.3<c_{m}\right)$, and higher than $c_{m},\left(3.7>c_{m}\right)$.

Case 3: $c_{m} \geq c_{b}$

The object speed and limits of $c$ are set to $V=2, c_{a}=2.2$ and $c_{b}=2.4$. Then $c_{m}$ $\approx 2.4495$. In Figure 3(a), $c$ is a single-valued monotonically decreasing function. In Figure 3(b) the PDF of $E$ again decreases monotonically when $c$ has the uniform distribution. Figure 3(c) shows three cases: the $c$-value of the vertex of the triangular probability distribution of $c$ is $2.25,2.3$ or 2.35 .

\section{Discussion}

When the speed of light is assumed to be variable, its probability distribution is unknown. Although this study only examines two probability distributions for it, the probability distribution of $E$ has certain characteristics. If the distribution of $c$ is uniform, the probability density of $E$ is always maximum at minimum $E$ and decreases monotonically regardless of whether $c_{m}$ is within the range of $c$ or not. If the distribution of $c$ is triangular and $c_{m} \leq c_{a}$, the distribution of $E$ is also triangle-like with the vertex moving rightward with $c$. If the distribution of $c$ is triangular and $c_{m} \geq c_{b}$, the probability distribution of $E$ changes from trapezoid-like shape to triangle-like shape. In both cases the vertex of the probability distribution of $E$ moves together with the probability distribution of $c$. In contrast with these cases, if $c_{a}<c_{m}<c_{b}$, the PDF of $E$ is always maximum at minimum $E$ in either distribution of $c$. This is because the PDF of $E$ diverges to infinity at minimum $E$ in either distribution of $c$ from Equation (14). In practice, the PDF of $E$ at minimum $E$ increases even faster as the calculation step becomes smaller. This equation shows that if $c_{a}<c_{m}<c_{b}$, the PDF of $E$ is always maximum at minimum $E: E=\sqrt{3} m_{0} c_{m}^{2}$ regardless of the distribution of $c$ (that is, diverges to infinity). This suggests that even if the distribution of $c$ is unknown, $E$ will rapidly increase as soon as $c_{m}$ enters a certain range of $c$ as the speed $V$ of an object increases.

On this basis, the following method is proposed to detect any range of $c$. As the speed of light is defined as $299,792,458 \mathrm{~m} / \mathrm{s} \equiv c_{L}, \quad c_{a} \leq c_{L} \leq c_{b}$.

If

$$
c_{m}=\frac{\sqrt{6}}{2} V<c_{a} \leq c_{L} \leq c_{b}
$$

then

$$
V<\frac{\sqrt{6}}{3} c_{L} .
$$

For example, the speed of one thousand electrons or protons is increased stepwise by an accelerator from below $\frac{\sqrt{6}}{3} c_{L}$ to $c_{L}$. For each step, a frequency 
distribution of $E$ will be obtained. As $V$ is increased from below $\frac{\sqrt{6}}{3} c_{L}, c_{m}$ will enter the range of $c$ at the critical value of $V$. Then the probability density of minimum $E$ will increase sharply. Since the speed of light has been measured with very fine precision [4], the range of $c$ would be very narrow. Then the speed will need to be more finely increased bit by bit (in steps of $100 \mathrm{~m} / \mathrm{s}$ if possible). As $V$ increases after $c_{m}$ exceeds $c_{b}$, the probability density of minimum $E$ will decrease abruptly. If these phenomena are observed, then $c$ is variable. If not, then $c$ is constant.

\section{Conclusion}

If it is possible that the speed of light in free space is variable, then a probabilistic method to detect the variability is applicable. This assumes that $c$ obeys a probability distribution. From mass-energy equivalence, the PDF of $E$ can be obtained using the inverse function of $E$. The energy is minimum at $c_{m}=\frac{\sqrt{6}}{2} V$, and the PDF of $E$ diverges to infinity at $E=\sqrt{3} m_{0} c_{m}^{2}$. Thus, when $c_{m}$ enters the range of $c$ as $V$ is increased stepwise from below $\frac{\sqrt{6}}{3} c_{L}$, the PDF of $E$ increases abruptly regardless of the PDF of $c$. If this is observed by accelerating a beam of electrons or photons, it will show that $c$ is variable; otherwise, $c$ is constant.

\section{Acknowledgements}

Mark Kurban, M.Sc., from Edanz Group (http://www.edanzediting.com/ac) edited a draft of this manuscript.

\section{Conflicts of Interest}

The author declares that there is no conflict of interests regarding the publication of this paper.

\section{References}

[1] Einstein, A. (1905) Ist die Trägheit eines Körpers von seinem Energieinhalt abhängig? Annalen der Physik, 323, 639-641.

https://doi.org/10.1002/andp.19053231314

[2] Michelson, A.A. and Morley, E.W. (1887) On the Relative Motion of the Earth and the Luminiferous Ether. American Journal of Science, 34, 333-345. https://doi.org/10.2475/ajs.s3-34.203.333

[3] Evenson, K.M., Wells, J.S., Petersen, F.R., Danielson, B.L. and Day, G.W. (1973) Accurate Frequencies of Molecular Transitions Used in Laser Stabilization: The 3.39- $\mu \mathrm{m}$ Transition in $\mathrm{CH}_{4}$ and the 9.33- and 10.18- $\mu \mathrm{m}$ Transitions in $\mathrm{CO}_{2}$. Applied Physics Letter, 22, 192-195. https://doi.org/10.1063/1.1654607

[4] Herrmann, S., Senger, A., Möhle, K., Nagel, M., Kovalchuk, E.V. and Peters, A. (2009) Rotating Optical Cavity Experiment Testing Lorentz Invariance at the $10-17$ Level. Physical Review D, 80, Article ID: 105011. https://doi.org/10.1103/PhysRevD.80.105011 
[5] Giovannini1, D., Romero1, J., Potoček, V., Ferenczi, G., Speirits, F., Barnett, S.M., Faccio, D. and Padgett, M.J. (2015) Spatially Structured Photons That Travel in Free Space Slower than the Speed of Light. Science, 347, 857-860.

https://doi.org/10.1126/science.aaa3035 